\title{
Calvarium Thinning in Patients with Spontaneous Cerebrospinal Fluid Leaks of the Anterior Skull Base \\ Running Head: Calvarium Thinning in sCSF-L Patients
}

Authors: Dhruv Sharma, MD; Brady J. Tucker, BS²; David S. Millay, OD²; Kolin E. Rubel, MD; Mohamad Saltagi, MD'; Rick F. Nelson, $\mathrm{MD}^{1}$; Elisa A. Illing, MD¹; Jonathan Y. Ting, MD, MS, $\mathrm{MBA}^{1}$

Author Affiliations: ${ }^{1}$ Department of Otolaryngology - Head and Neck Surgery, Indiana University, Indianapolis, IN; ${ }^{2}$ Indiana University School of Medicine, Indianapolis, IN

\section{Corresponding Author:}

Dhruv Sharma, MD

Department of Otolaryngology - Head and Neck Surgery

Indiana University School of Medicine

1130 W. Michigan Street, Suite 400, Indianapolis, IN 46202

Telephone: 317-278-1286 | Fax: 317-274-8285 | E-mail: dhsharm@iu.edu

Presentation: Accepted for Presentation at the American Rhinologic Society Annual Meeting to be held virtually September $11-12^{\text {th }}, 2020$.

Conflict of Interest: None

Funding: None

Manuscript Word Count: 2976

This is the author's manuscript of the article published in final edited form as:

Sharma, D., Tucker, B. J., Millay, D. S., Rubel, K. E., Saltagi, M., Nelson, R. F., Illing, E. A., \& Ting, J. Y. (2021). Calvarium Thinning in Patients with Spontaneous Cerebrospinal Fluid Leaks of the Anterior Skull Base. The Laryngoscope, 131(6), 1271-1276. https://doi.org/10.1002/lary.29130 


\section{ABSTRACT}

Objective: Patients with spontaneous cerebrospinal fluid leaks (sCSF-L) of the temporal bone have isolated calvarial and skull base thinning that is independent of obesity. The study determines if anterior skull base (ASB) SCSF-L patients also have calvarial thinning.

Methods: Retrospective cohort study of ASB sCSF-L patients compared to non-obese (body mass index, $\mathrm{BMI}<30 \mathrm{~kg} / \mathrm{m}^{2}$ ) and obese (BMI $\geq 30$ ) control groups. 21 patients in the ASB SCSF-L group and 25 patients in each control group were included. Calvarium and extracranial zygoma thicknesses were measured bilaterally with blinded, standardized volumetric analysis. Results: ASB sCSF-L patients had a mean [SD] age of 50.43 [10.19] years, an average [SD] BMI of 38.81 [8.92] kg/m², and most were female (85.71\%). The calvarium in patients with ASB SCSF-L was significantly thinner than the non-obese $(2.55 \mathrm{~mm}[0.77]$ vs. $2.97[0.67] \mathrm{mm} ; P=$ $0.006 ; 95 \% \mathrm{Cl}, 0.12$ to 0.30 ; Cohen $d, 0.58)$ and obese control groups (2.55 [0.77] vs. 2.92 $[0.76] \mathrm{mm} ; P=.02 ; 95 \% \mathrm{Cl}, 0.05$ to 0.34 ; Cohen $d, 0.66)$. The calvarium thickness of the nonobese patients was not significantly different from the obese patient controls $(2.97$ [0.67] vs. $2.92[0.76] \mathrm{mm}, P=0.9)$. The extracranial zygoma was not significantly different among the groups (ANOVA, $P=0.33$ ).

Conclusions: ASB SCSF-L patients have isolated calvarial thinning that is independent of obesity. Like lateral skull base SCSF-L patients, these data suggest that additional obesityassociated intracranial process contributes to skull thinning.

Abstract Word count: 240/250

Key words (3-7): Anterior Skull Base, Computed Tomography, CSF Rhinorrhea, Endoscopic Skull Base Surgery, Skull Base Repair

Level of Evidence: 4 


\section{Introduction}

Spontaneous cerebrospinal fluid leaks (sCSF-L) may develop when there is a defect in the bone of the anterior or lateral skull base (ASB, LSB) with interruption of its accompanying arachnoid and dural layers without an inciting factor. Non-spontaneous causes include a preceding trauma, benign or malignant tumors, or an iatrogenic cause such as skull base surgery. ${ }^{1,2}$ ASB sCSF-L typically present with persistent unilateral rhinorrhea and anosmia, whereas LSB defects in the temporal bone commonly present with persistent middle ear effusion with associated conductive hearing loss. ${ }^{3}$

While often presenting with benign symptoms, these defects provide a conduit linking the intracranial space to the external environment and harbor the potential for serious complications such as meningitis, stroke, and death. The possibility of severe complications in SCSF-L combined with a significant rise in incidence has driven multiple specialties to investigate the underlying etiology and pathophysiology of SCSF-L, in an attempt to potentially prevent its development or recurrence. ${ }^{4,5}$

Studies have found that SCSF-Ls are increasingly common and most prevalent in middle-aged, obese females. ${ }^{6}$ The incidence of surgical repair of LSB SCSF-L doubled between 2002 to 2012 , which was consistent with the growing obesity epidemic in the United States. ${ }^{5}$ While obesity has strongly correlated with SCSF-Ls, recent studies suggest that this strong association may be driven by obesity-related sequelae including obstructive sleep apnea (OSA) and elevated intracranial pressure (ICP) that lead to skull base thinning, dehiscence, and finally SCSF-L. ${ }^{2,7-10}$ However, it remains unknown whether SCSF-L patients have inherently thinner bone or if obesity-related sequelae are truly causing erosion of the skull base and calvarium.

It is known in the neurotology literature that patients with LSB sCSF-Ls have global calvarial thinning, and recent studies have found that OSA and ICP are both independently associated with thinning of the calvarium. ${ }^{8-10}$ With regards to the ASB, Psaltis et al. reported that SCSF-L patients had thinning in the region of the ethmoid roof, lateral lamella, and anterior face 
of the sella compared with controls and traumatic leak patients. ${ }^{11}$ In this retrospective casecontrol study, the authors hypothesize that the same pathologic process that leads to erosion of the ASB also causes global calvarium thinning in SCSF-L patients.

\section{Materials and Methods}

\section{Patient Selection}

The study was approved by the institutional review board at Indiana University School of Medicine (protocol \# 1912413724). A retrospective study design was utilized. Data was abstracted from the electronic medical record (EMR) system for our tertiary care referral center from January 2015 to October 2019. All patients with ASB sCSF-Ls that were surgically repaired during this time period were included in the study group. Inclusion criteria for the control groups included (1) high-resolution maxillofacial computed tomography (CT) imaging (2) patients age 18 or older and less than 80 years. Patient at the extremities of age were excluded, as agerelated changes in bone thickness may artificially change cortical thickness measurements in control groups, based on anecdotal institutional data.

In order to capture the study population of interest, patients who had ASB surgery were identified using the following surgical Current Procedural Terminology codes: 31290 (nasal/sinus endoscopy, surgical, with repair of CSF-L; ethmoid), 31291 (nasal/sinus endoscopy, surgical, with repair of CSF-L; sphenoid), 31299 (unlisted procedure, accessory sinuses), and 64999 (unlisted procedure, nervous system). The charts of 269 identified patients were manually reviewed to determine inclusion and exclusion from the study group. Exclusion criteria included: (1) history of functional endoscopic sinus surgery (FESS), ASB surgery other than SCSF-L repair, radiation to head and neck, and facial trauma, and (2) primary surgery involving the resection of a sinonasal tumor. The medical records of 29 patients who had a surgical repair of an ASB SCSF-L by two fellowship trained rhinologists were reviewed, and 8 patients were eliminated because the CT imaging was either inaccessible or inadequate for analysis (>2-mm 
axial and coronal sections). In summary, 11 patients who had CT maxillofacial imaging with a thickness of $1-\mathrm{mm}$ and 10 patients who had one with a thickness of $2-\mathrm{mm}$, for a total of 21 patients in the ASB sCSF-L group, were included in the study.

In the control groups, patients with OSA were excluded as the disease process has been found to be independently associated with calvarial thinning. ${ }^{2,8}$ The sleep disorders center database at Indiana University was utilized to identify all patients who underwent a formal diagnostic polysomnogram (PSG) with an apnea-hypopnea index ( $\mathrm{AHI}$ ) less than 5 and also met the imaging criteria (167 patients). SCSF-L patients were excluded by chart review. By separating these patients by body mass index (BMI), 80 non-obese patients $(\mathrm{BMI}<30 \mathrm{~kg} / \mathrm{m} 2)$ and 87 obese patients $(\mathrm{BMI} \geq 30)$ were identified. Within these two groups, 25 patients were randomly selected for inclusion in order to adequately power the study. The EMR for control group patients was reviewed to confirm that each one did not have a history of elevated ICP or a diagnosis of idiopathic intracranial hypertension, which have been found to be independently associated with calvarial thinnning. ${ }^{7}$ All patients in the control groups had imaging with $\leq 1$-mm axial and coronal sections. In summary, patients were divided into the following 3 groups: 1) surgical cohort of ASB sCSF-L patients $(n=21) 2)$ non-OSA $(A H I<5)$, non-obese group $(B M I<$ 30) control group $(n=25)$, and 3) non-OSA, obese group (BMI > 30) control group $(n=25)$.

Demographic data, including age, gender, race, were obtained for all three groups. Race data were obtained and assigned from the electronic medical record as documented during previous patient hospital visits; therefore, the authors had no role in classifying race. BMI at time of referenced imaging study was also obtained. In the study group, a diagnosis of OSA confirmed by PSG or history of continuous positive airway pressure (CPAP) use was documented.

\section{Radiologic Analysis and Measurements}

After the patient groups were finalized, CT scans were obtained, deidentified, and randomized for analysis. Measurements were performed by two evaluators who were blinded to 
group status associated with the CT scan. Radiologic measurements were performed using 3D Slicer (version 4.6.2, http://www.slicer.org), which is an open source, NIH-funded image analysis tool. An accurate and consistent method of measuring calvarial and zygoma thickness had previously been developed and utilized at our institution by Rabbani et al, and the same methodology was utilized. ${ }^{8}$ For practical purposes, the squamous portion of the temporal bone was measured at its thinnest sections and these data were extrapolated to global calvarium thickness (Figure 1A and B). Measurements were performed and reported bilaterally, with $n=$ number of measurements, yielding 2 measurements for each patient.

Calvarial thickness was measured beginning just anterior to the foramen rotundum in the coronal plane: 1) Measurements began by selecting and highlighting $15 \mathrm{~mm}$ (in height) of the thinnest part of the calvarium within $4 \mathrm{~cm}$ of the skull base. A threshold of 400 Hounsfield units was applied for highlighting bone. 2) Moving in a posterior direction, $15 \mathrm{~mm}$ (in height) portions of bone were sequentially highlighted while avoiding air cells, posteriorly to the level of the superior extent of the superior semicircular canal (SCC). 3) This length of bone measurement was calculated using distances provided by 3D slicer. 4) Volumes were obtained of the highlighted bone using 3D slicer's volume analysis tool. 5) Measurements were performed bilaterally, yielding a total of 142 measurements $(\mathrm{N}=142)$ on 71 patients. 6$)$ With the volume, length, and height known, the following calculation was applied to determine the average calvarial thickness on each side: Calvarial Thickness = Volume / [Height $x$ Length]. These values were averaged to obtain the overall average calvarial thickness.

The extracranial zygoma was then measured with the same 400 Hounsfield unit threshold along a $5 \mathrm{~mm}$ anterior-posterior segment of the zygomatic process just anterior to its attachment to the temporal bone, as seen on coronal images. The zygoma was measured as an internal imaging control as it is a consistent extracranial bone in all images. After highlighting the appropriate number of slices needed to reach approximately $5 \mathrm{~mm}$ in length, the volume was obtained. Length was calculated by multiplying the number of slices by the thickness of each 
slice, and this value was approximately $5 \mathrm{~mm}$ consistently. Finally, by treating our coronal zygoma cross-sections as a cylinder, we were able to use the following formula to determine zygoma thickness: Zygoma thickness $=2 *(\sqrt{ }($ Volume $/($ length $* \pi))$.

\section{Statistical Analysis}

Statistical analysis was performed using SPSS statistical software (version 24, IBM Corp) to determine the existence of a significant difference between measurements. Two-tailed independent sample $t$ tests were applied in comparing the calvarial thickness between groups, and $95 \%$ confidence intervals $(\mathrm{Cl})$ were used to inform statistical and clinical significance. Cohen $d$ was used to measure effect size when indicated with $d=0.2$ to be a small effect, $d=$ 0.5 to be a moderate effect, and $d \geq 0.8$ to be a large effect. An ANOVA test was utilized to compare all 3 groups when appropriate.

\section{Results}

21 ASB SCSF-L patients were evaluated and compared to 25 patients in each nonobese and obese control group. The complete demographics and clinical data are presented in Table 1. In comparing the BMI for SCSF-L and obese groups, there was no statistically significant difference (two-tailed t-test, $p=0.31$ ). There was no statistically significant difference in age among the three groups (ANOVA, $p=0.23$ ). There were a majority of females in all three groups $(85.7 \%, 68.0 \%, 76.0 \%$, respectively) with no statistically significant difference in gender (ANOVA, $p=0.38$ ). All groups were predominantly white $(76.2,76$, and $68.0 \%$, respectively) with no statistically significant difference in race (ANOVA, $p=0.77$ ). In the study group, one patient (4.6\%) had PSG-confirmed OSA (AHI of 11), six patients (28.6\%) had a diagnosis of OSA in the EMR, and no patients had a record of using CPAP. All control patients from the two control groups had PSG test that demonstrated no evidence of OSA. Although nineteen patients (90.5\%) had lumbar drains placed for surgery, only four of those patients (19.1\%) had a documented ICP with a mean (standard deviation [SD]) opening pressure of 36.7 [13.9] $\mathrm{cm} \mathrm{H}_{2} 0$. 
After the lumbar drain was clamped post-operatively, nine patients (42.9\%) had a documented ICP that was measured $\left(37.8 \mathrm{~cm} \mathrm{H}_{2} \mathrm{O}[8.6]\right)$. In the 13 patients that had documented ICP intraoperatively or postoperatively, 11 patients $(84.6 \%)$ had evidence of intracranial hypertension.

Since measurements were conducted bilaterally on CT imaging, 42 measurements in the ASB SCSF-L group and 50 measurements in each control group were performed. The squamous temporal bone was first measured in the study population with ASB sCSF-Ls to determine the calvarium thickness. When comparing patients who had CT imaging with 1-mm thick sections $(n=11)$ to patients who had CT imaging with 2-mm thick sections $(n=10)$, there was no significant difference in mean (range, SD) calvarial thickness $(2.35 \mathrm{~mm}$ [1.35-4.00, 0.81] vs. $2.77[1.86-4.05,0.68] ; p=0.08)$. Next, the SCSF-L group $(n=21)$ was compared to the nonOSA group with a $\mathrm{BMI}<30$. When compared to non-obese controls, the mean calvarial thickness in ASB SCSF-L patients was significantly thinner $(2.55 \mathrm{~mm}[1.35-4.05,0.77]$ vs. 2.97 $[1.81-4.83,0.67] ; p=0.006 ; 95 \% \mathrm{Cl}, 0.12$ to 0.30 ; Cohen $d, 0.58$; Figure 2A). The mean difference was $0.42 \mathrm{~mm}$, which represents a $15.2 \%$ thinner calvarium.

Next, the authors sought to determine if obesity was independently associated with thinner of the calvarium. To this end, the squamous temporal bone was measured in non-OSA patients with a $\mathrm{BMI} \geq 30$. There was no significant difference in mean (SD) calvarium thickness between the non-obese and obese control groups $(2.97[1.81-4.83,0.67]$ vs. $2.92[1.58-4.88$, $0.76] ; p=0.72 ; 95 \% \mathrm{Cl},-0.23$ to $0.34 ;$ Cohen $d, 0.10$; Figure $2 A)$. Furthermore, the mean calvarial thickness of ASB sCSF-L patients was significantly thinner when compared to the obese control group $(2.55[1.35-4.05,0.77]$ vs. $2.92[1.58-4.88,0.76] ; p=0.02 ; 95 \% \mathrm{Cl}, 0.05$ to 0.34; Cohen d, 0.66; Figure 2A). The mean difference was $0.37 \mathrm{~mm}$, which represents a $13.5 \%$ thinner calvarium. As an extracranial control, the mean (SD) zygoma thickness was measured in each group, and there was no significant difference among the SCSF-L, non-obese, and obese groups $(5.07$ [3.54-6.94, 0.89] vs. $4.88[3.46-6.42,0.84]$ vs. $5.16[3.36-7.85,1.08]$; 
ANOVA, $p=0.33$; Figure 2B). Within the ASB sCSF-L group, there was also no significant difference in the zygoma thickness between patients with 1 -mm thick sections vs. 2-mm (4.86 $[3.59-6.57,0.83]$ vs. $5.29[3.67-6.94,0.93] ; p=0.12)$.

\section{Discussion}

This study demonstrates that patients with ASB sCSF-Ls have a significantly thinner calvarium compared to non-obese and obese control groups, indicating that calvarial thinning in this cohort is independent of obesity. Nelson et al reported that there was significant thinning of the calvarium by $23 \%$ in patients who underwent MCF approach for surgical repair of LSB SCSF-L compared to age-matched non-obese and obese cochlear implant control groups. ${ }^{2,9}$ In the ASB SCSF-L population, we found that the calvarium was $13.5-15.2 \%$ thinner. Therefore, the results of the two studies are consistent, suggesting there is global calvarial thinning in SCSF-L patients regardless of location of the leak (ASB versus LSB) and independent of BMI. The authors here also confirm that calvarial thinning is isolated to the intracranial bone, since there were no significant differences in extracranial zygoma thickness among patients in the three studied groups. These results suggest against a systemic bone metabolism condition (e.g. osteoporosis) as the cause of global calvarial thinning. Instead, our data indicate that an intracranial process is thinning the calvarium of ASB SCSF-L patients. Considering the thickness of both the anterior and lateral skull bases are typically less than $1 \mathrm{~mm}$, global thinning of the calvarium and skull base of 0.6 to $1.0 \mathrm{~mm}$ secondary to an intracranial process can lead to dehiscence of the skull base and CSF leak. ${ }^{12,13}$ We postulate that the mechanisms and pathophysiology responsible for this calvarial thinning in ASB SCSF-Ls patients overlap with the thinning observed in patients with leaks of the LSB. ${ }^{2,9}$

The endeavor to understand the etiology of SCSF-L has been an ongoing pursuit dating back to the 1960s, with Ommaya et al discussing non-traumatic cerebrospinal fluid leaks (CSFL) and increased intracranial pressure (ICP). ${ }^{14}$ Multiple retrospective studies, systematic reviews, 
and meta-analyses have established relationships between SCSF-L and several clinical factors, which include obesity, female gender, middle age, idiopathic intracranial hypertension (IIH), and obstructive sleep apnea (OSA)..$^{6-10,14-16}$ Patients with SCSF-Ls are on average 45 to 65 years old, predominantly female with a 7:3 female to male ratio, and obese with an average BMI ranging from 35 to $38 \mathrm{~kg} / \mathrm{m}^{2} .{ }^{4}$ The patients in our surgical cohort were consistent with the literature with an average $\mathrm{BMI}$ of 39 , although there was a slightly higher prevalence of females $(86 \%)$ compared to the literature on ASB SCSF-L patients $(77 \%) .{ }^{4}$ It is important to note that gender was previously found to have no effect on mean calvarial or zygoma thickness. ${ }^{9}$

Obese patients have a greater risk for developing and a higher prevalence of OSA and $\mathrm{IIH} .{ }^{12}$ Both of these comorbid conditions are believed to have an indirect and direct connection to elevated ICPs. ${ }^{2,15,17}$ Transient or chronic increases in ICP are thought to play a significant role in the development of SCSF-Ls by exerting mechanical pressure-like forces on the skull base and calvarium, which cause thinning of the bone as well as exposure and tearing of the dural layers. Furthermore, elevated ICPs and thinner skull bases have been associated with higher rates of postsurgical complications. ${ }^{18,19}$

OSA has been independently connected with both calvarial thinning and SCSF leaks.,20 The etiology behind this phenomenon is believed to stem from transient spikes in intracranial pressure observed during apneic episodes. ${ }^{2}$ Interestingly, although only one patient in our ASB SCSF-L cohort had PSG-confirmed OSA, six (28.6\%) had a clinical diagnosis of OSA. This is similar to the rate previously published in one retrospective study which showed that the prevalence of OSA in ASB SCSF-L patients was 30.0\% compared to $14.3 \%$ in their nonspontaneous counterparts. ${ }^{1}$ Interestingly, this study was included in a systematic review of retrospective studies, which reported an overall $16.9 \%$ incidence of SCSF-L patients having OSA. ${ }^{14}$ Their meta-analysis showed that SCSF-L patients had a 4.73 and 2.85 times more likely chance of having OSA compared to control cohorts and nonspontaneous CSF-L patients, respectively. ${ }^{14}$ Lobo et al. reported in another systematic review that $43-45 \%$ of ASB and LSB 
SCSF-L patients had a diagnosis of OSA on presentation. ${ }^{4}$ In a prospective cohort of LSB sCSF$\mathrm{L}$ patients, the prevalence of OSA was nearly twice as high at $83.3 \% .{ }^{20}$ In a similar fashion, the prevalence of OSA in the ASB population may be significantly higher than currently reported in the literature. Therefore, prospective studies utilizing PSGs are required to determine the true prevalence of OSA in the ASB SCSF-L population.

Intracranial hypertension $(\mathrm{IH})$ is another complication frequently noted in both obese individuals and individuals with OSA. ${ }^{7}$ Similar to OSA, IH has been associated with skull base thinning in ASB sCSF-Ls patients. ${ }^{2,7,11}$ More specifically, Rabbani et al showed that IH was independently associated with both global calvarium and skull base thinning. ${ }^{10}$ Moreover, BMI has been shown to be directly correlated with increasing CSF pressures and displays a direct linear relationship. ${ }^{2}$ Another study showed a dose-response effect of increasing BMI with the risk of developing $\mathrm{IIH} .{ }^{21}$ Furthermore, numerous studies have reported elevated ICP in patients with ASB SCSF-Ls with one study reporting an average opening pressure of $33 \mathrm{~cm} \mathrm{H} \mathrm{H}_{2} \mathrm{O}$, and two prospective series have demonstrated a 7.6 to $8.0 \mathrm{~cm} \mathrm{H}_{2} \mathrm{O}$ increase in ICP following surgical repair of SCSF-L. ${ }^{22-24}$ In our study, 11 of 13 patients (84.6\%) who had a documented ICP intraoperatively or postoperatively had evidence of intracranial hypertension. Interestingly, this falls above the wide range of 10 to $66 \%$ of SCSF-L patients who were found to have elevated ICPs in the literature, which is likely secondary to the small sample size of patients who had a documented ICP in our study group..$^{25-27}$

There are limitations to this study that warrant discussion. The small sample size of 21 ASB SCSF-L patients is certainly a limitation, though the strict imaging criteria which excluded 8 patients was important for consistency. Since there is twice the potential for error with 2-mm thick sections, we confirmed there was no significant difference in calvarial and zygoma thickness within the ASB sCSF-L cohort for patients with CT sections of 1-mm versus 2-mm. However, there was certainly a trend with the 2-mm thick CTs averaging higher thickness than 1-mm. This suggests that if all patients within the ASB sCSF-L group had 1-mm thick CTs then 
the difference in calvarial thickness may have been greater with the study population's calvaria averaging even lower. Future studies would benefit from using multi-institutional data for a larger sample size with the utilization of $\leq 1 \mathrm{~mm}$ thick imaging.

Overall, the authors confirm that a large majority of patients with ASB sCSF leaks are obese, middle-aged females, which is consistent with the existing literature. Furthermore, these data demonstrate global calvarial thinning in ASB SCSF-L patients that is independent of obesity, showing that thinning is not only isolated to the anterior skull base as previously reported. ${ }^{11}$ Although obesity is known to correlate strongly with SCSF-Ls, it is likely the obesity-related comorbidities that lead to global calvarium thinning and skull base dehiscence.

\section{Conclusion}

ASB sCSF-L patients have global calvarial thinning that is independent of BMI. Obesityrelated comorbidities, including OSA and IIH, likely independently contribute to an intracranial process which causes both skull base and calvarial thinning. The underlying etiologies and pathophysiology of SCSF-L continue to warrant further investigation. 


\section{References}

1. Fleischman GM, Ambrose EC, Rawal RB, et al. Obstructive sleep apnea in patients undergoing endoscopic surgical repair of cerebrospinal fluid rhinorrhea. Laryngoscope. 2014;124(11):2645-2650.

2. Rabbani CC, Saltagi MZ, Nelson RF. The role of obesity, sleep apnea, and elevated intracranial pressure in spontaneous cerebrospinal fluid leaks. Curr Opin Otolaryngol Head Neck Surg. 2019;27(5):349-355.

3. Naples JG, Shah RR, Ruckenstein MJ. The evolution of presenting signs and symptoms of lateral skull base cerebrospinal fluid leaks. Curr Opin Otolaryngol Head Neck Surg. 2019;27(5):344-348.

4. Lobo BC, Baumanis MM, Nelson RF. Surgical repair of spontaneous cerebrospinal fluid (CSF) leaks: A systematic review. Laryngoscope Investig Otolaryngol. 2017;2(5):215-224.

5. Nelson RF, Gantz BJ, Hansen MR. The rising incidence of spontaneous cerebrospinal fluid leaks in the United States and the association with obesity and obstructive sleep apnea. Otol Neurotol. 2015;36(3):476-480.

6. Perani D, Scotti G, Colombo N, et al. Spontaneous CSF rhinorrhea through the lamina cribrosa associated with primary empty sella. Ital J Neurol Sci. 1984;5(2):167-172.

7. Rabbani CC, Patel JM, Nag A, et al. Association of Intracranial Hypertension With Calvarial and Skull Base Thinning. Otol Neurotol. 2019;40(6):e619-e626.

8. Rabbani C, Saltagi MZ, Ye MJ, et al. Association of Obstructive Sleep Apnea With Calvarial and Skull Base Thinning. JAMA Otolaryngol Head Neck Surg. 2018;144(6):513518.

9. Nelson RF, Hansen KR, Gantz BJ, Hansen MR. Calvarium thinning in patients with spontaneous cerebrospinal fluid leak. Otol Neurotol. 2015;36(3):481-485.

10. Bakhsheshian J, Hwang MS, Friedman M. Association Between Obstructive Sleep Apnea and Spontaneous Cerebrospinal Fluid Leaks: A Systematic Review and Meta-analysis. JAMA Otolaryngol Head Neck Surg. 2015;141(8):733-738.

11. Psaltis AJ, Overton LJ, Thomas WW, 3rd, et al. Differences in skull base thickness in patients with spontaneous cerebrospinal fluid leaks. Am J Rhinol Allergy. 2014;28(1):e73-79.

12. Gottlieb DJ, Punjabi NM. Diagnosis and Management of Obstructive Sleep Apnea: A Review. Jama. 2020;323(14):1389-1400.

13. Kennedy DW, Hwang PH. Rhinology. Diseases of the Nose, Sinuses, and Skull Base.

14. Bidot S, Levy JM, Saindane AM, et al. Do Most Patients With a Spontaneous Cerebrospinal Fluid Leak Have Idiopathic Intracranial Hypertension? J Neuroophthalmol. 2019;39(4):487-495.

15. Martinez-Capoccioni G, Serramito-Garcia R, Martin-Bailon M, et al. Spontaneous cerebrospinal fluid leaks in the anterior skull base secondary to idiopathic intracranial hypertension. Eur Arch Otorhinolaryngol. 2017;274(5):2175-2181.

16. Ganjaei KG, Soler ZM, Mappus ED, et al. Novel Radiographic Assessment of the Cribriform Plate. Am J Rhinol Allergy. 2018;32(3):175-180. 
17. O'Connell BP, Stevens SM, Xiao CC, et al. Lateral Skull Base Attenuation in Patients with Anterior Cranial Fossa Spontaneous Cerebrospinal Fluid Leaks. Otolaryngol Head Neck Surg. 2016;154(6):1138-1144.

18. Stevens SM, Rizk HG, Mcllwain WR, et al. Association between Lateral Skull Base Thickness and Surgical Outcomes in Spontaneous CSF Otorrhea. Otolaryngol Head Neck Surg. 2016;154(4):707-714.

19. Yancey KL, Manzoor NF, Kelly PD, et al. Impact of obesity and obstructive sleep apnea in lateral skull base cerebrospinal fluid leak repair. Laryngoscope. 2019.

20. Rabbani CC, Saltagi MZ, Manchanda SK, et al. Prevalence of Obstructive Sleep Apnea (OSA) in Spontaneous Cerebrospinal Fluid (CSF) Leaks: A Prospective Cohort Study. Otol Neurotol. 2018;39(6):e475-e480.

21. Daniels AB, Liu GT, Volpe NJ, et al. Profiles of obesity, weight gain, and quality of life in idiopathic intracranial hypertension (pseudotumor cerebri). Am J Ophthalmol. 2007;143(4):635-641.

22. Chaaban MR, Illing E, Riley KO, Woodworth BA. Acetazolamide for high intracranial pressure cerebrospinal fluid leaks. Int Forum Allergy Rhinol. 2013;3(9):718-721.

23. Chaaban MR, Illing E, Riley KO, Woodworth BA. Spontaneous cerebrospinal fluid leak repair: a five-year prospective evaluation. Laryngoscope. 2014;124(1):70-75.

24. Wang EW, Zanation AM, Gardner PA, et al. ICAR: endoscopic skull-base surgery. Int Forum Allergy Rhinol. 2019;9(S3):S145-s365.

25. Allen KP, Perez CL, Kutz JW, et al. Elevated intracranial pressure in patients with spontaneous cerebrospinal fluid otorrhea. Laryngoscope. 2014;124(1):251-254.

26. Brainard L, Chen DA, Aziz KM, Hillman TA. Association of benign intracranial hypertension and spontaneous encephalocele with cerebrospinal fluid leak. Otol Neurotol. 2012;33(9):1621-1624.

27. Kim L, Wisely CE, Dodson EE. Transmastoid approach to spontaneous temporal bone cerebrospinal fluid leaks: hearing improvement and success of repair. Otolaryngol Head Neck Surg. 2014;150(3):472-478. 


\section{Figure Legends:}

Figure 1: Abbreviations: mm, millimeters. Calvarial and Zygoma Measurement Methodology: A) Coronal maxillofacial CT showing a representative calvarial measurement of a 15-mm (height) segment of the thinnest portion of the squamous temporal bone (green). The extracranial zygoma was measured as an extracranial control (purple). B) 3-Dimensional reconstruction illustrating the measured $15 \mathrm{~mm}$ segment of calvarium (green) and $5 \mathrm{~mm}$ segment of zygoma (purple).

Figure 2: Abbreviations: NS, not significant; sCSF-L, spontaneous cerebrospinal fluid leak. Calvarial and Zygoma Thickness in BMI $<30, \mathrm{BMI} \geq 30$, and SCSF-L Patients: A) sCSF-L patients had significantly thinner calvaria compared to both control groups. Calvarial thickness was not significantly different between $\mathrm{BMI}<30$ and $\mathrm{BMI} \geq 30$ patients. B) There were no statistically significant differences in zygoma thickness among the three study groups. 
Table 1: Patient Demographics and Clinical Characteristics

\begin{tabular}{|c|c|c|c|}
\hline & $\begin{array}{c}\text { sCSF-L } \\
\mathbf{( n = 2 1 )}\end{array}$ & $\begin{array}{c}\text { Non-Obese Control } \\
\mathbf{( n = 2 5 )}\end{array}$ & $\begin{array}{c}\text { Obese Control } \\
\mathbf{( n = 2 5 )}\end{array}$ \\
\hline Age, mean (Range, SD), y & $50.4(32.0-72.0,10.2)$ & $54.2(29.0-77.0,10.9)$ & $55.8(40.0-78.0,10.9)$ \\
\hline BMI, mean (Range, SD), y & $38.8(27.1-62.6,8.9)$ & $25.3(18.9-29.3,2.8)$ & $36.4(42.0-78.0,6.5)$ \\
\hline Gender, No (\%) & & & $19(76.0)$ \\
\hline Female & $18(85.7)$ & $17(68.0)$ & $6(24.0)$ \\
\hline Male & $3(14.3)$ & $8(32.0)$ & $17(68.0)$ \\
\hline Race, No (\%) & & & $7(28.0)$ \\
\hline White & $16(76.2)$ & $19(76.0)$ & $1(4.0)$ \\
\hline Black & $5(23.8)$ & $6(24.0)$ & $0(0.0)$ \\
\hline Hispanic & $0(0.0)$ & &
\end{tabular}

Abbreviations: SCSF-L, spontaneous cerebrospinal fluid leak; SD, standard deviation; $y$, years;

BMI, body mass index (calculated as weight in kilograms divided by height in meters squared) 